\title{
A Mechanistic and Stochastic Approach to Fatigue Crack Nucleation in Coarse Grain RR1000 Using Local Stored Energy
}

Authors: Yan Bin Pan $^{1, *}$, Prof. Fionn P.E. Dunne ${ }^{1}$, Dr. Duncan W. MacLachlan ${ }^{1,2}$

1 Department of Materials, Imperial College London, South Kensington Campus, London, SW7 2AZ 2 Rolls-Royce plc, PO Box 31, Derby, DE24 8BJ, United Kingdom

* Corresponding author, yan.pan14@alumni.imperial.ac.uk

\begin{abstract}
The crystal plasticity finite element (CPFE) method is used in conjunction with a critical local stored energy criterion to predict crack nucleation life for Coarse Grain (CG) nickel superalloy RR1000. Artificial representative microstructures are generated using Dream3D, and through simulation of multiple microstructural instantiations a distribution of simulated fatigue response is generated. Fatigue of CG RR1000 is studied at 300 and $700^{\circ} \mathrm{C}$ and at two R-ratios of R=0.1 and $\mathrm{R}=-1$ giving a range of conditions to test the stored energy method. At higher temperature failure frequently occurs from inclusions, these are represented in the model by adding an inclusion with cohesive zones between inclusion and matrix. The results at $300^{\circ} \mathrm{C}$ are very good with the one parameter model (the critical stored energy) able to predict the mean, slope and distribution of fatigue data. At $700^{\circ} \mathrm{C}$ the results are also good, however fatigue life at high strain amplitude is over-predicted.
\end{abstract}

\section{Keywords:}

Crystal Plasticity, Fatigue Life Prediction, Monte Carlo Method, non-metallic inclusion, Nickel based Superalloy, Crack Nucleation.

\section{Nomenclature:}

$\begin{array}{llll}b & \text { Burger's vector } & \dot{p} & \text { Plastic strain rate } \\ E & \text { Young's modulus } & \dot{\gamma}^{\alpha} & \text { Shear strain rate } \\ \boldsymbol{F}^{\mathrm{E}} & \text { Elastic deformation } & \Delta F & \text { Helmholtz constant } \\ \boldsymbol{F}^{\mathrm{P}} & \text { Plastic deformation } & \Delta G_{S} & \text { Stored energy increment per cycle } \\ \boldsymbol{F}^{\mathrm{T}} & \text { Total deformation } & \Delta V & \text { Activation volume } \\ G_{1} & \text { Shear modulus } & \xi & \text { Percentage of plastic work stored in the material }\end{array}$




$\begin{array}{llll}G_{C} & \text { Critical stored energy } & \lambda & \text { Hardening coefficient } \\ G_{i} & \text { Initial stored energy } & \nu & \text { Frequency dislocation escape } \\ \kappa & \text { Boltzman constant } & \rho_{S S d m} & \text { Mobile statistically stored dislocations density } \\ \boldsymbol{L}^{\mathrm{P}} & \text { Velopcity gradient } & \dot{\rho}_{S S D} & \text { Evolution of statistically stored dislocations } \\ \mathbf{n}^{\alpha} & \text { Normal slip direction } & \tau_{c 0} & \text { Initial critical resolved shear stress } \\ \mathbf{s}^{\alpha} & \text { Shear slip direction } & \tau_{c}^{\alpha} & \text { Critical resolved shear stress } \\ N_{f} & \text { Nucleation life } & & \end{array}$

\section{Introduction}

The last decade has seen a significant escalation in the combined use of advanced microstructural modelling and high-resolution characterization techniques. The level of validation of microstructurally explicit Crystal Plasticity Finite Element (CPFE) methods used for simulating deformation at the scale of individual grains and below, is such that industrial use of these methods has become quantitative and tractable. Validation of CPFE models using characterization data from techniques such as HR-EBSD (High Resolution Electron Back Scattered Diffraction) and HR-DIC (Digital Image Correlation) $)^{4,5}$ mean the internal distributions of stresses and strains, caused by the heterogeneity of microstructural features, are known to be simulated to a high degree of accuracy, such that they can be used as a basis for calculating progressive damage and crack nucleation. To take advantage of these developments it is timely to test the range of applicability of microstructure based modelling methods, and to test their ability to predict databases of fatigue behavior over a range of load conditions. This is an essential stage of their development to more general use in industrial design and structural integrity assessment. Foremost amongst the benefits of microstructurally based fatigue modelling is its potential to simulate distributions of fatigue life. Safety critical aero-engine components are cleared for service by taking account of the distribution of fatigue life and such that the probability of a crack initiating over the entire service life of a fleet of components is remotely small. It is invariably therefore the tail of the fatigue distribution that is used to clear component lives, which is difficult and costly to determine through mechanical testing. Conservative estimates of fatigue life are frequently used in the aerospace industry as a result of both the criticality of the components as well as the uncertainty in the underlying distribution. From an industrial perspective therefore, a key objective of microstructural fatigue modelling is to be able to predict fatigue distributions through modelling the different levels of microstructural heterogeneity that cause them, such as weak and strong grain orientations, inclusions, precipitates, twins and 
boundaries ${ }^{6}$.

The link between the stochastic nature of fatigue and microstructural features has been recognized for many years. Early work by Sauzay and Jourdan ${ }^{7}$ showed the relationship between the scatter in fatigue life data and the distribution of resolved shear stress in different crystallographic orientations in Copper and Austenitic metals. They showed the distribution of internal stresses is largely responsible for determining the distribution of fatigue scatter. Sangid et al. ${ }^{8}$ used energetic calculations of Persistent Slip Band (PSB) formation using molecular dynamic simulations (Sangid et al. ${ }^{9,10}$ ) to develop a stochastic model for fatigue of nickel Udimet 720Li. Nucleation life was correlated with PSB formation and the distribution was derived by running multiple simulations on artificial but representative grain structures. Other researches have also developed the principle of determining fatigue distributions by running multiple instances of artificial microstructures, Stopka and McDowell ${ }^{11}$, Tang Gu et al. ${ }^{12}$, Gillner and Munstermann ${ }^{13}$, Owolabi and Whitworth ${ }^{14}$.

The link between plastic deformation, slip and the generation of dislocation structures is well established, although the appropriate mechanism and formulation used to relate these phenomena to fatigue life is not, perhaps as a result of different mechanisms dominating nucleation in different structures. Mughrabi ${ }^{15}$ addressed the importance of the formation of dislocation substructures on cyclic hardening using SEM observation of PSB formation. The effect of PSB formation on crack nucleation was also addressed by Dunne et al. ${ }^{16}$. Using CPFE to explicitly model surface-PSB matrix interaction Repetto and Ortiz ${ }^{17}$ showed the crack nucleation life could be predicted by the time taken for the subtended angle of the extruded PSB with the material surface to reach zero, thus forming a sharp crack. Dislocation-density based methods have also been used, Shanthraj and $\mathrm{Zikri}^{18}$, that have related high grain boundary dislocation densities to intergranular and transgranular fracture. Mughrabi ${ }^{19}$ analysed the effect of cyclic slip irreversibility, by partitioning total plastic strain into reversible and irreversible components for different dislocation interactions they derived a Coffin-Manson style relation using irreversible plastic strain instead of total plastic strain. Manonukul and Dunne ${ }^{20}$ working on nickel base alloy C263 demonstrated the ability of total accumulated slip to predict crack nucleation in both the LCF and HCF regimes. With continued increases in computational capacity discrete dislocation dynamics is becoming more and more widespread and has been used to provide fundamental links between crack nucleation and dislocation configuration, Zheng et al. ${ }^{21}$, Prastiti et al. ${ }^{22}$, see Lavenstein and El-Awady ${ }^{23}$ for a review.

As it is clearly not yet possible to design components using dislocation based models (although in specific circumstances design rules can be derived from such fundamental models, Zheng et al. ${ }^{24}$ ) a 
link is still required between sub-grain dislocation based models of crack nucleation and microstructural level models used to quantify the role of microstructure quantities on crack formation. In a similar way to the development of continuum level Fatigue Indicator Parameters (FIPs), most notably that of Fatemi-Socie ${ }^{25}$, since the development of the CPFE method the applicability of a range of microstructural FIPs for correlating and predicting fatigue life has been investigated for a number of materials. Dong-Feng Li et al. ${ }^{26}$ used a FIP based on accumulated plastic strain but modified for triaxiality and temperature effects to predict Thermo-Mechanical Fatigue (TMF) for P91 steels. Yuang et al. ${ }^{27}$ used accumulated plastic slip to predict crack nucleation in a range of sizes of micro-notch specimens in nickel based alloy GH4169. More recently the same author $^{28}$, also working on GH4169 found the dissipated energy was more accurate at high strain amplitudes than the accumulated plastic strain. Dabiri et al. ${ }^{29}$ used total strain in conjunction with critical distance theory to predict notch fatigue crack nucleation and showed that a microstructural approach was more accurate than conventional methods, this work was on a high strength steel. Owalabi and Whitworth ${ }^{14}$ used the maximum plastic shear strain range to predict nucleation and combined it with a local Fatemi-Socie parameter to predict short crack growth in coarse grain nickel alloy IN 100. Gillner and Munstermann ${ }^{13}$, based on the work of Musinski and McDowell ${ }^{30}$ studied a ferrite-pearlite steel and used the grain averaged maximum plastic strain as a FIP and also ran many models to develop an extreme value statistical correlation for high cycle fatigue life. Bridier and McDowell $^{31}$ carried out an investigation using a 3D CPFE model to study grain scale plastic deformation for a Ti-6Al-4V titanium alloy. It showed how at low cyclic strains single slip is predominantly promoted through a softening evolution law for each slip system.

A proposal to unify the various micro-mechanical quantities that have been used to model crack nucleation was made ${ }^{1-2,4-5}$ in the definition of the critical stored energy criterion. This quantity is the energy retained in the material resulting from plastic work and accumulated shear deformation on different active systems. The hypothesis is that when localized cyclic plasticity occurs, some of the plastic energy generated is dissipated as heat and the remainder goes into the formation of dislocations and eventually networks of dislocation structures. With repeated cycling these dislocation structures become more refined until they cause sufficient hardening to result in local cleavage of the material and to nucleate a fatigue facet. The area over which the energy is calculated is given by the mean free spacing between dislocations which provides a fundamental length scale for the nucleation process. Wan et al. ${ }^{1}$ analyzed a series of body centered cubic, large grained ferritic steel, notched bend fatigue test samples. CPFE models of the exact grain morphologies and orientations were used to determine dislocation densities, accumulated plastic slip and slip rate. The 
results showed that the local stored energy gave the best estimates of both the location of crack nucleation and the number of cycles to nucleation across all the test data. In later work Chen et al. ${ }^{4}$, studying bend fatigue in single and oligo superalloy crystals showed that whilst slip, stress and Geometrically Necessary Dislocation (GND) density were significant parameters, only the local stored energy predicted nucleation in all tests. Subsequently, Chen et al. ${ }^{5}$ investigating powder Ni alloy FGH96, used HR-DIC and HR-EBSD to quantitatively calculate local stresses, strains and dislocation densities at a number of nucleation sites around a large inclusion. Again, they found local stored energy gave the best correlation with nucleation life. Since this work discrete dislocation analyses ${ }^{21,22}$ have successfully compared the local stored energy to the dislocation configurational energy providing a fundamental link from the microstructural level (stored energy) to the sub-grain level (configurational energy).

A key aim of this work is to further test the applicability of the local stored energy parameter for prediction/correlation of fatigue lives and to determine if it can provide a link between fundamental modelling techniques and engineering application. An additional aim is to quantify fatigue scatter using a Monte Carlo simulation approach where multiple microstructural instantiations are run at each test condition such that a fatigue distribution can be generated entirely by simulation. Using this approach, the ability of the CPFE modelling method combined with the local stored energy nucleation parameter, is tested to predict the distribution of fatigue life rather than just the mean. Whilst the results at lower temperatures of $300^{\circ} \mathrm{C}$ are very good the method over-predicts the lives of the fatigue test data at the highest strain level at $700^{\circ} \mathrm{C}$. It is thought that this is due to crack nucleation being dominated by oxide inclusions in the PM (Powder Metallurgy) alloy at high temperature.

\section{Methods}

\subsection{Material and Test Data}

The material under investigation in this work is Coarse Grain (CG) RR1000. CG RR1000 is a powder processed, $\gamma^{\prime}$ precipitation hardened superalloy, its chemical composition is given in Table 1. The heat treatment for this material consists of a supersolvus solution and aging treatment resulting in a coarse grain microstructure with an average grain size of approximately 30 microns. CG RR1000 has the same chemical composition as RR1000 but a different heat treatment designed to ensure a larger grain size and therefore a higher temperature capability than fine grained RR1000. 
Fatigue test data for this project was kindly provided by Rolls-Royce plc. In all cases the fatigue data is for strain control plain specimen testing with no stress concentration features. The fatigue test piece drawing used in the testing is shown in figure 1. The specimen surfaces were peened before testing which was performed at two temperatures of $300^{\circ} \mathrm{C}$ and $700^{\circ} \mathrm{C}$ and two R-ratios (ie. min. strain/max. strain) of $R=0.1$ and $R=-1$.

Table 1. Nominal composition of CG RR1000

\begin{tabular}{|l|l|l|l|l|l|l|l|l|l|l|l|}
\hline & Ni & Co & $\mathrm{Cr}$ & $\mathrm{Mo}$ & $\mathrm{Ti}$ & $\mathrm{Al}$ & $\mathrm{Ta}$ & $\mathrm{Hf}$ & $\mathrm{Zr}$ & $\mathrm{C}$ & $\mathrm{B}$ \\
\hline Wt\% & Bal. & 18.5 & 15.0 & 5.0 & 3.6 & 3.0 & 2.0 & 0.5 & 0.06 & 0.02 & 0.03 \\
\hline At\% & Bal. & 17.9 & 16.5 & 3.0 & 4.3 & 6.35 & 0.63 & 0.16 & 0.04 & 0.14 & 0.1 \\
\hline
\end{tabular}

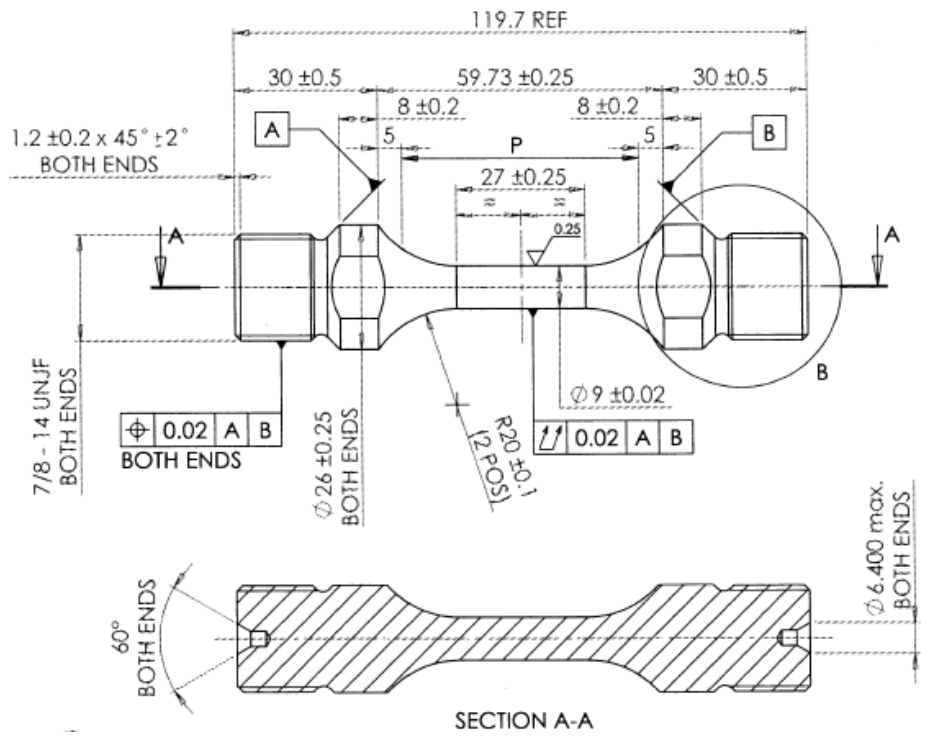

Figure 1. Test piece drawing for fatigue tests performed on CG RR1000.

\subsection{Crystal Plasticity Model and Calibration}

This section describes the slip rule and hardening variables that are used in the CPFE model. The FE implementation of the crystal plasticity model used is similar to that described in Wan et al ${ }^{1,2}$ and Chen et al. ${ }^{4,5}$ and only the essential details are repeated here. A comprehensive review and analysis of the fundamental mechanical aspects of crystal plasticity can be found in Khan and Huang ${ }^{32}$. $\mathrm{Ni}$ 
based CG RR1000 has Face Centered Cubic (FCC) symmetry and therefore 12 primary octahedral slip systems of type $\{111\}<110\rangle$ that can be activated during loading. These are the only systems that are represented in the current model. Whilst it is acknowledged that other systems, such as cubic $\{100\}<011>$ systems, or type II octahedral systems $\{111\}<112>$, can be activated at high temperatures and certain orientations, the majority of deformation at the temperatures considered occurs on type I systems. The crystal plasticity model is rate dependent and is implemented as a user material (UMAT) subroutine for the ABAQUS finite element program.

The standard crystal plasticity approach is used where the total deformation gradient $\boldsymbol{F}^{\mathrm{T}}$ is decomposed into the elastic $\boldsymbol{F}^{\mathrm{E}}$ and plastic $\boldsymbol{F}^{\mathrm{P}}$ deformation gradients ${ }^{1-2,32}$

$$
\boldsymbol{F}^{\mathrm{T}}=\boldsymbol{F}^{\mathrm{E}} F^{\mathrm{P}}
$$

The velocity gradient is obtained by summing the individual shear strains occurring on active systems $^{1-2,32}$

$$
\boldsymbol{L}^{\mathrm{P}}=\dot{\boldsymbol{F}}^{\mathrm{P}} \boldsymbol{F}^{\mathrm{P}-1}=\sum^{12} \dot{\gamma}^{\alpha} \mathbf{s}^{\alpha} \otimes \mathbf{n}^{\alpha}
$$

The sum $\alpha$ is over the 12 type 1 octahedral systems, $\dot{\gamma}^{\alpha}$, is the slip system shear strain rate, $\mathbf{s}^{\alpha}$ and $\mathbf{n}^{\alpha}$ are the unit slip system direction and slip plane normal vectors respectively and $\otimes$ denotes outer product. The slip rule used for each of the active slip systems in the model is ${ }^{1-2,4-5}$

$$
\dot{\gamma}^{\alpha}=\rho_{s s d m} b^{2} v \exp \left(-\frac{\Delta F}{k T}\right) \sinh \left(\frac{\Delta V}{\kappa T}\left|\tau^{\alpha}-\tau_{c}^{\alpha}\right|\right)
$$

Numerical values and units of all physical quantities are given in table 2. $\rho_{s s d m}$ is the mobile dislocation density, $b$ is the magnitude of the Burgers vector, $v$ the frequency of dislocation attempts to jump energy barriers considering both forward and backward jumps, $\Delta F$ the activation energy, $\Delta V$ the associated activation volume, $\kappa$ the Boltzmann constant, $T$ the temperature, $\tau^{\alpha}$ the resolved shear stress for slip system $\alpha$ and $\tau_{c}^{\alpha}$ is the corresponding critical resolved shear stress. A dislocation based hardening rule is used to determine the evolution of the critical resolved shear stress $\tau_{c}^{\alpha} 1,2$

$$
\tau_{c}^{\alpha}=\tau_{c 0}+G_{12} \cdot b \cdot \sqrt{\rho_{s s d}+\rho_{g n d}}
$$

where $\tau_{c 0}$ is the critical resolved shear stress and $G_{12}$ is the in-plane shear modulus, $b$ is the magnitude of the Burgers vector and $\rho_{s s d}$ and $\rho_{g n d}$ are the SSD and GND densities

The evolution of dislocation density in terms of accumulated slip rate $\dot{p}$ is given by ${ }^{1,2}$ 


$$
\dot{\rho}_{S S D}=\lambda \cdot \dot{p}
$$

where $\lambda$ is the hardening coefficient. $\dot{p}$ is the equivalent plastic strain rate that has been defined $\operatorname{as}^{20}$

$$
\dot{p}=\left(\frac{2}{3} L^{\mathrm{P}}: \boldsymbol{L}^{\mathrm{P}}\right)^{1 / 2}
$$

Equation 6 is a convenient large strain measure of the effective plastic deformation rate and is derived from its similarity to the conventional definition of effective, or equivalent plastic strain. The symbol (:) denotes the double contracted product of two second order tensors.

The CPFE model is calibrated to the correct macroscopic plastic response using a 3D cuboidal RVE with 200 grains. Linear hexahedral elements were used to construct the RVE. A fixed displacement of zero was applied to the bottom $(\mathrm{Z}=0)$ surface of the cube whilst the necessary displacement to generate the required overall macro strain was applied to the opposite face of the cube. The displacement in all cases was applied as a ramp with 1 second duration to match the test data. Planar restraints are applied to the sides of the RVE such that no relative out-of-plane deformation between nodes on the external surface is allowed. Results for fatigue 'hotspots' later in the work are only investigated away from the RVE free surface such that the exact surface boundary condition used doesn't affect the results. Details of the microstructure RVE generation process are described in the next section. Sufficient grains must be simulated to ensure the macro response is invariant of the random grain orientations used for an RVE. Sensitivity studies using different numbers of grains showed the variability in macro-response reduced to $2 \%$ when 200 grains were used which was deemed to be a sufficiently accurate and as a result 200 grains are used for all RVEs in this work. Figure 2 shows the calibrated elastic-plastic model plotted against the experimental response at $300^{\circ} \mathrm{C}$ and $700^{\circ} \mathrm{C}$. All the model parameters used are tabulated in table 2.
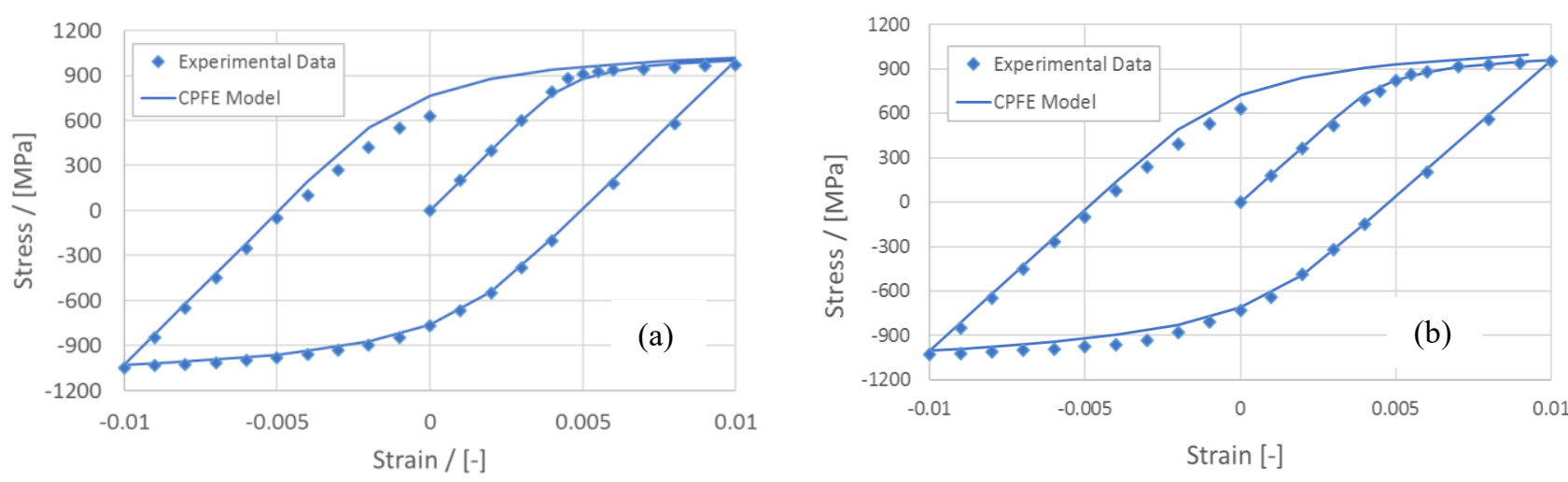

Figure 2. Calibrated model and experimental stress-strain response for first cycle full hysteresis loop at (a) $300^{\circ} \mathrm{C}$ and (b) $700^{\circ} \mathrm{C}$. 
Table 2. CPFE calibrated model parameters at $300^{\circ} \mathrm{C}$ and $700^{\circ} \mathrm{C}$.

\begin{tabular}{|c|c|c|c|}
\hline Temp & ${ }^{\circ} \mathrm{C}$ & 300 & 700 \\
\hline$\tau_{c 0}$ & $\mathrm{MPa}$ & 300 & 280 \\
\hline$\lambda$ & $\mu \mathrm{m}^{-2}$ & 50 & 50 \\
\hline$\rho_{s s d m}$ & $\mu \mathrm{m}^{-2}$ & 0.05 & 0.05 \\
\hline$v$ & $\mathrm{~s}^{-1}$ & $1 \mathrm{e}^{11}$ & $1 \mathrm{e}^{11}$ \\
\hline$\kappa$ & $\mathrm{JK}^{-1}$ & $1.381 \mathrm{e}-23$ & $1.381 \mathrm{e}-23$ \\
\hline$\Delta F$ & $\mathrm{~J} / \mathrm{Atom}$ & $3.46 \mathrm{e}-20$ & $3.46 \mathrm{e}-20$ \\
\hline$b$ & $\mu \mathrm{m}$ & $3.51 \mathrm{e}-4$ & $3.51 \mathrm{e}-4$ \\
\hline$\rho_{S S D}$ & $\mathrm{~m}^{-2}$ & $1 \mathrm{e}^{10}$ & $1 \mathrm{e}^{10}$ \\
\hline$\Delta V$ & {$[-]$} & $8.54 \mathrm{~b}^{3}$ & $8.54 \mathrm{~b}^{3}$ \\
\hline$E$ & $\mathrm{GPa}$ & 127.5 & 109.3 \\
\hline$G_{I 2}$ & $\mathrm{GPa}$ & 121.0 & 105.3 \\
\hline$n u$ & {$[-]$} & 0.39 & 0.41 \\
\hline & & & \\
\hline
\end{tabular}

\subsection{RVE Model Generation and Monte Carlo (MC) Analysis}

An interesting feature of CG RR1000 is the large variation in grain size where grains can vary from approximately $2 \mu \mathrm{m}$ up to $150 \mu \mathrm{m}$ with an average of $30 \mu \mathrm{m}$. Dream $3 \mathrm{D}^{3}$ software is used to generate representative grain geometries for CPFE models with a grain size distribution similar to that of $\mathrm{CG}$ RR1000. Figure 3 shows a comparison of the actual and simulated (Dream3D representation) grain size distribution. The grain size distribution in Dream3D uses a lower and upper limit of $0.22 \mu \mathrm{m}$ and $113.2 \mu \mathrm{m}$ respectively. There is no preferred material orientation so a random texture distribution was used. Figure 4(a) shows an example model created by Dream3D for this microstructure with a hexahedral element mesh. Figure 4(b) shows the pole figures for the random texture distribution. Using an RVE with 200 grains and an average diameter of $30 \mu \mathrm{m}$ results in overall cube edge dimensions of $174 \mu \mathrm{m}$. We have not in this work explicitly accounted for the presence of annealing twins in the microstructure. Whilst it is well known that twins can play a key role in determining nucleation life ${ }^{35}$ the extra level of mesh refinement required to model sub-grain features has precluded their inclusion in a Monte Carlo analysis such as this. Whilst twins have an important impact on nucleation it is likely to be a relative effect, such that the overall conclusions regarding the relative strengths at different loads, temperatures and R-ratios in the absence of twins is still valid. 


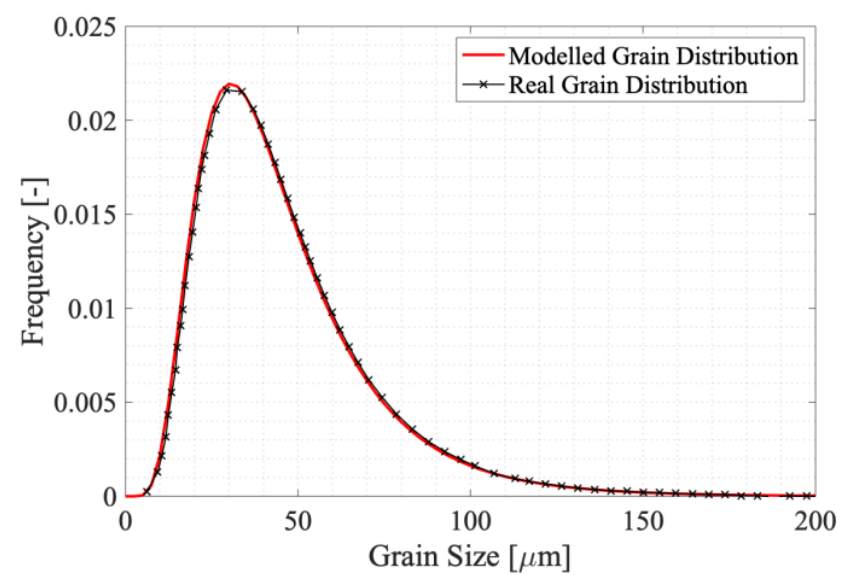

Figure 3. Actual grain size distribution and simulated distribution generated by Dream3D.
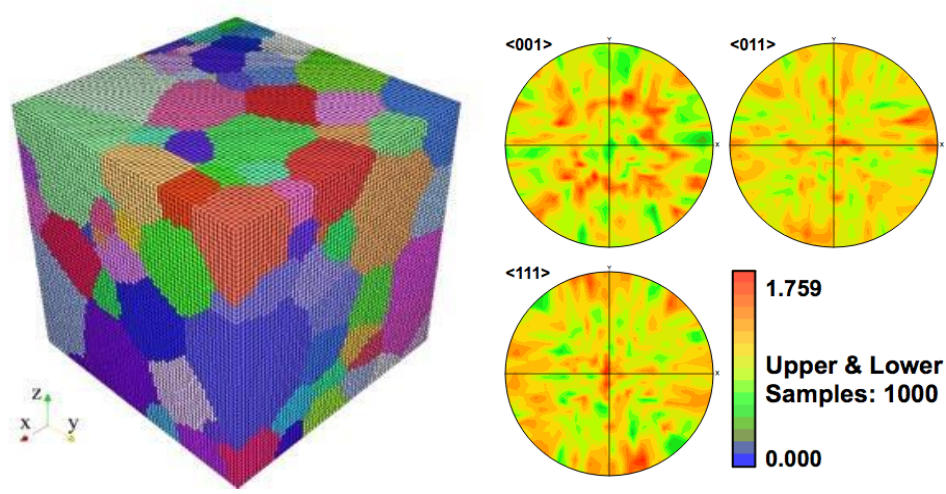

(a)

(b)

Figure 4. (a) Surface grain morphology with hex element mesh. (b) Pole figure of the CPFE model.

A regular gridded hexahedral mesh composed of three dimensional eight noded linear C3D8 elements as shown in figure 4(a) was used in all analyses. A mesh refinement study was conducted to determine the required number of elements to give converged quantities with a reasonable computational time. The analysis found that approximately 200,000 elements was sufficient to accurately capture local stress and strain gradients.

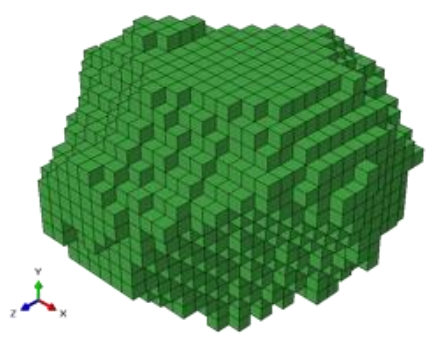

Figure 5. Example of a single isolated grain generated using a Dream3D hexahedral mesh. 
Each microstructural CPFE fatigue model is used to simulate a single fatigue life at a particular test condition; however it is not known a priori where in the distribution of fatigue lives that this point will lie. As a result a number of CPFE models must be run to establish the mean and distribution of the population of fatigue lives. If this approach is successful it will test a key requirement of microstructural modelling which is to predict distributions in fatigue data resulting from variations in microstructure. Clearly many CPFE simulations are required to test this fully and rigorously for extreme tails. Computational time restricted the number of simulations that could practically be run in this initial investigation, so we are therefore limited to testing the principle. For each of the conditions at which experimental mechanical fatigue data are available five separate microstuctural instantiations were run. Under the two temperatures and two R-ratios investigated in this work, a total of 20 different test conditions were available (there are multiple experimental test results at many of these conditions) and therefore 100 simulations were run which is a significant computational outlay, with each simulation taking $\sim 15$ hours to run. Each of the 100 simulations used a different granular RVE microstructure and random orientation distribution.

As it is required to build a large number of CPFE models a Matlab Graphical User Interface (GUI) was developed to automate the process. The GUI is essentially a wrapper for Dream3D. A single Dream3D pipeline is built which contains all the microstructural parameters required to generate a representative CG RR1000 microstructure. A picture of the main GUI window is shown in figure 6 below. The user is asked to choose the appropriate pipeline (in our case CG RR1000) and then, in the main tabular area in the bottom half of the window, all the relevant test conditions are entered, such as stress/strain control, maximum and minimum loadings and temperature. There is also the ability to enter more complicated test waveforms as well as TMF and multiaxial loadings. A key feature of the GUI is that for each set of test conditions entered the user can select how many microstructural instantiations are to be run, in this work we have used five at each condition. Finally, the number of actual fatigue cycles required to be run in the FE model can be specified. In these analyses we have set this to five cycles which is sufficient to stabilize the cyclic response, this is discussed further in the next section. 


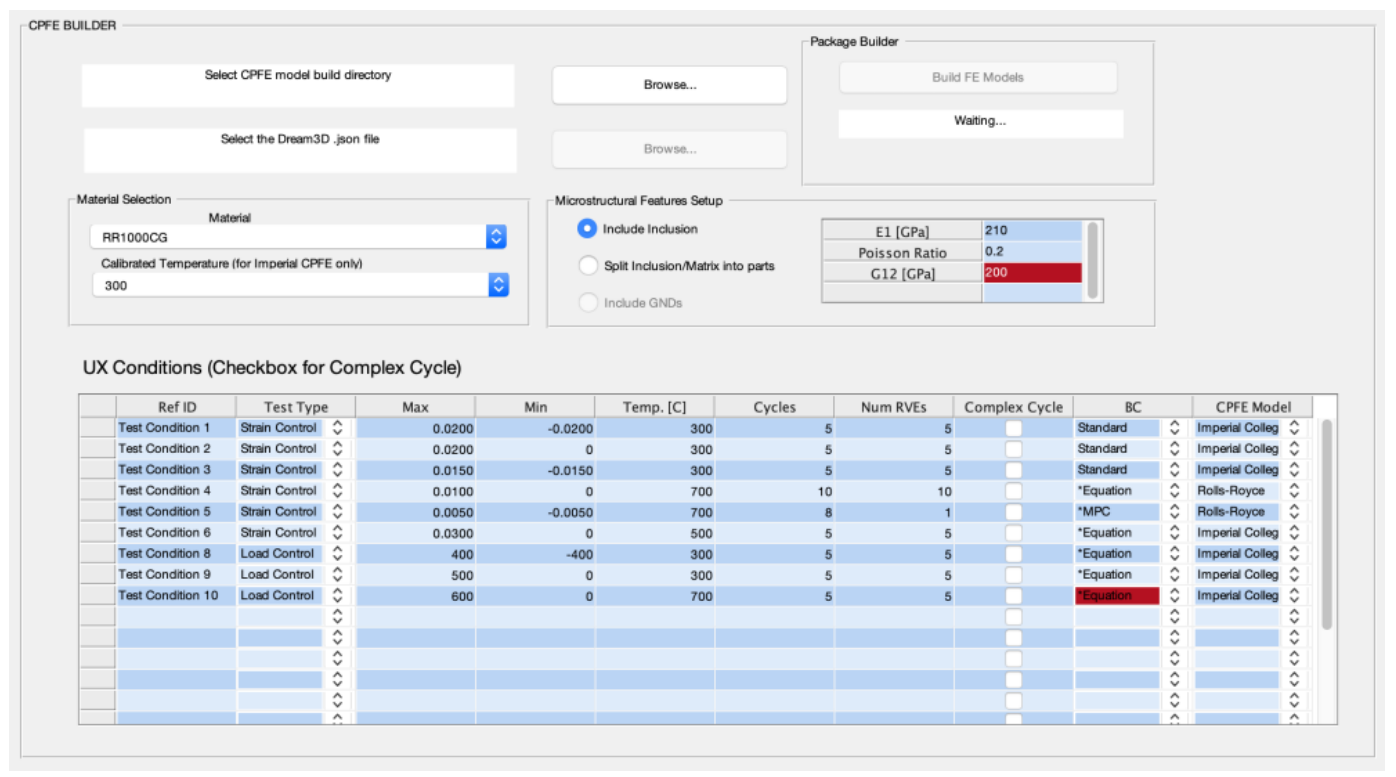

Figure 6. Matlab GUI used to automate construction of multiple CPFE RVEs.

\subsection{Hard Inclusion and Cohesive Zone Modelling}

Under some conditions, particularly at high temperature, fatigue cracks in CG RR1000 can nucleate from internal oxide inclusions. This mechanism was modelled explicitly in a series of CPFE simulations by embedding an inclusion within the microstructure. The size and shape of inclusion particles varies significantly but they are frequently similar in size to the grain size of the alloy studied. For simplicity therefore, to model the effect of an inclusion, a single grain at the centre of an RVE is identified and its single-crystal properties are replaced with those of the isotropic oxide. Representative oxide properties of $400 \mathrm{GPa}$ for Young's Modulus and $160 \mathrm{GPa}$ for shear modulus were used to define the elastic behaviour of the inclusion. As the tests and simulations are at elevated temperature the thermal mismatch between the alloy and the inclusion needs to be accounted for. The isotropic thermal expansion coefficients used for the alloy and inclusion are respectively $1.4 \mathrm{e}-5$ and $9.1 \mathrm{e}-6$ per ${ }^{\circ} \mathrm{C}$ at $700^{\circ} \mathrm{C}$.

To define the interface between the nickel matrix and inclusion a bilinear uncoupled tractionseparation relation is used in ABAQUS and its properties defined through damage initiation and damage evolution parameters. An uncoupled relationship between normal and shear traction is used since we expect the normal traction to be the primary driver of decohesion as demonstrated by Zhang et $\mathrm{al}^{33}$ and Bergsmo and Dunne ${ }^{37}$. In these studies decohesion between the metal-oxide interface and oxide particle fracture are observed experimentally and shown to correlate with high interfacial 
normal stresses and maximum principal stress normal to the fractured plane respectively. Also, the significant stress redistribution that occurs once the oxide agglomerate matrix interface decohere is shown to significantly change the energy distribution local to the oxide particle. Our analysis models the redistribution of energy by explicitly incorporating cohesive zone between oxide agglomerate matrix interface, as this will affect the mechanistic driver of crack nucleation. Therefore, we can infer that the tangential and transverse shear behaviour play only a minor role in the decohesion and particle fracture process. The elastic behaviour of the cohesive zone, before damage initiation, is determined by a stiffness matrix that relates the normal and shear stresses to the normal and shear displacements and defined via $K_{n n}, K_{s s}$ and $K_{t t}$, where the subscripts $n, s$ and $t$ refer to the normal and two shear components. The damage initiation criterion used is that separation can occur when the surface tractions reach their critical values $\mathrm{t}_{n}{ }^{o}, \mathrm{t}_{s}{ }^{o}, \mathrm{t}_{t}{ }^{o}$. Material data for the metal-oxide interface was obtained from Bergsmo and Dunne ${ }^{37}$. This work argued that the process of decohesion is stress controlled and that failure occurs in a brittle manner releasing all its traction after reaching a peak value. Thus, the choice of a linear traction-separation relation is justified as the shape of the curve would be of secondary importance only. As the traction quickly drops to zero upon unloading convergence issues are encountered due to the large stiffness changes. To mitigate this effect a viscous coefficient of 0.01 has been chosen according to ${ }^{38}$. Table 3 tabulates all the parameters used in the analysis.

Table 3. Cohesive surface parameters used in the CPFE analysis ${ }^{37}$.

\begin{tabular}{|c|c|c|}
\hline Parameter & Units & Value \\
\hline$K_{n n}$ & $\mathrm{GPa}$ & 190 \\
\hline$K_{s s}$ & $\mathrm{GPa}$ & 190 \\
\hline$K_{t t}$ & $\mathrm{GPa}$ & 190 \\
\hline $\mathrm{t}_{n}{ }^{o}$ & $\mathrm{MPa}$ & 1800 \\
\hline $\mathrm{t}_{s}{ }^{o}$ & $\mathrm{MPa}$ & 1800 \\
\hline $\mathrm{t}_{t}{ }^{o}$ & $\mathrm{MPa}$ & 1800 \\
\hline$\delta_{f}^{0}$ & $\mu m$ & 1 \\
\hline Viscosity & {$[-]$} & 0.01 \\
\hline
\end{tabular}




\subsection{Calculation of Fatigue Nucleation Life}

The fatigue nucleation life is calculated as the number of cycles for the largest local stored energy, $G$, anywhere in the microstructure, to reach its critical value $G$ c. The development and continuous refinement of dislocation structures leads to progressively higher localized stress states which eventually become sufficient to result in local cleavage and nucleation of a small fatigue facet. We infer that the energy associated with slip is related to the energy of formation of the dislocation structures and therefore relate the stored energy at the local microstructural length scale to build up of local stresses and facet nucleation.

The local stored energy per cycle has been derived ${ }^{1}$ as

$$
\dot{G}=\oint_{C} \frac{\xi \sigma: d \varepsilon^{P}}{\sqrt{\rho_{s s d}}}
$$

Where $\xi$ is 0.05 and reflects the component of plastic work stored in the material as opposed to that dissipated by heat, $\boldsymbol{\sigma}$ and $d \boldsymbol{\varepsilon}^{P}$ are the stress tensor and the tensor of plastic strain increments respectively. A key factor in the calculation of a local energy is the area over which it is stored, and this length-scale is provided by the mean free distance between dislocations in equation 7. A nonlocal volume average of the stored energy is also used to reduce any mesh dependency in the model. The criterion for facet nucleation is simply when the local stored energy reaches the critical value denoted $G_{C}$.

As only five fatigue cycles were run in each of the CPFE simulations we need to establish if an approximate method can be obtained for calculating $\dot{G}$ in equation 7 throughout the entire fatigue life. Figure 7 shows the magnitude of the total stored energy increment per cycle in a number of CPFE models, all taken at the location of maximum stored energy in the model. The results are for the five different RVE instantiations at the maximum strain level tested at $700^{\circ} \mathrm{C}, \mathrm{R}=0.1$, for figure 7(a) and $\mathrm{R}=-1$ for figure 7(b). The five lines in each figure therefore represent evolution of maximum local energy in each of the five different RVEs run at the conditions indicated. In all cases the magnitude of the local energy increment drops sharply over the first three cycles. This is perhaps to be expected as the loading is sufficient to cause significant plasticity on the first cycle, followed by rapid stabilization due to a combination of stress redistribution and plastic hardening. In all cases the increment of stored energy is approaching stabilization after five cycles. As a result, therefore, partly due to the need to run so many CPFE simulations (100), five cycles were deemed sufficient to obtain an approximate value of the stabilized cyclic stored energy rate, $\dot{G}$. The final crack nucleation criteria used is when $G$ reaches the critical value of $G_{c}$ where $G$ is defined by 


$$
G=G_{C}=G_{i}+\left(N_{f}-5\right) \dot{G}
$$

Where $G_{i}$ and $\dot{G}$ are the initial stored energy accumulated after the first five cycles and the stabilized value of cyclic energy at the fifth cycle respectively. It is interesting to note from figure 7 that the effect of microstructural variation on the fatigue simulations is already apparent. The shakedown profiles of all the energy plots are similar but differ in magnitude. The slightly different values of stabilized energy increment at the fifth cycle, though small, when repeated over many cycles will lead to a significant stochastic variation in life calculation for each of the simulations. It is also noted that at the same maximum strain amplitude the $\mathrm{R}=-1$ tests give a significantly increased level of cyclic energy over the $\mathrm{R}=0.1$ tests due to the increased level of plastic hysteresis.

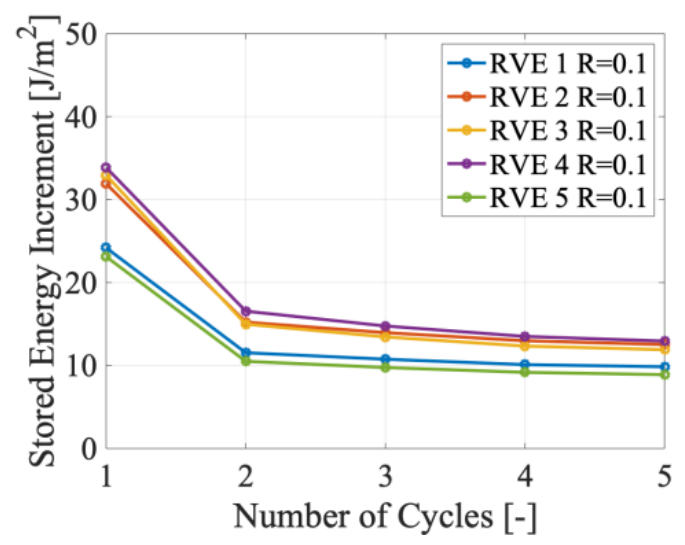

(a)

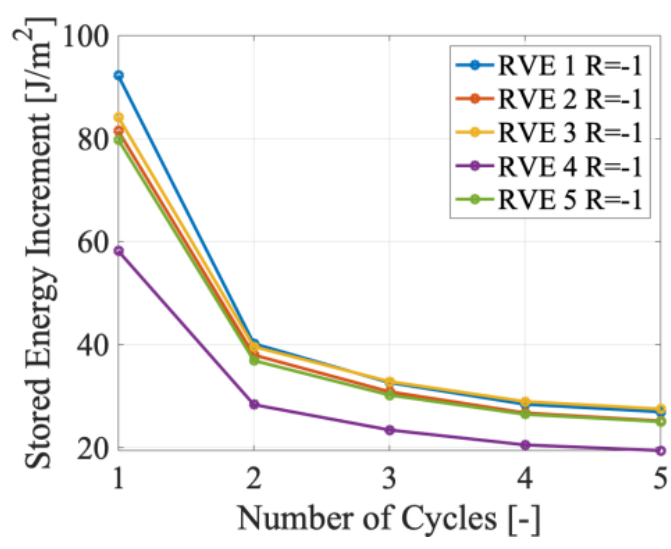

(b)

Figure 7. Stored energy increment over five cycles at the point of maximum stored energy for five different $\mathrm{CPFE}$ instantiations at $700^{\circ} \mathrm{C}$, (a) $\mathrm{R}=0.1$, (b) $\mathrm{R}=-1$.

It is important to note that predictions of life described here are only for the prediction of the nucleation of a localized fatigue facet of the order of microns in dimension. The model described cannot predict the initiation of a relatively large engineering crack of the order of 100s of microns in size. The separate terms nucleation and initiation are used to refer to nucleation of a very small fatigue facet and initiation of an engineering size fatigue crack (although it is recognized the size of an engineering crack is also variable, ideally this is the size where crack growth rate is no longer dependent on microstructure and/or the Paris Law can be used to predict crack growth rates). In section 3 the predicted facet nucleation lives are compared to mechanical test data where the failure life is actually for a $10 \%$ load drop and therefore for initiation of a significant crack. It is recognized that this is a significant limitation of the current approach and two efforts are underway to address this situation. The first is that focused small scale testing will be performed such that the nucleation 
life can be measured directly and the second is the development of a microstructurally explicit short crack growth model which will predict the life of crack growth from a nucleated facet to an initiated engineering crack.

\section{Results and Discussion}

In this section results are presented comparing fatigue test data with Monte-Carlo CPFE predicted nucleation lives where the life has been calculated using the method outlined in section 2.5.

\subsection{CPFE Model Predictions at $300^{\circ} \mathrm{C}$}

In figure 8 Monte Carlo (MC) CPFE predictions of fatigue crack nucleation are plotted against plain specimen fatigue life data at $300^{\circ} \mathrm{C}$ for both $\mathrm{R}=0.1$ and $\mathrm{R}=-1$. The critical stored energy, derived using the method outlined in section 2.5 by optimizing to match the experimental data across all strain levels and both R-ratios is $60000 \mathrm{Jm}^{-2}$. The $\mathrm{R}=0.1$ data is predicted accurately across all strain levels apart from the lowest of $0.5 \%$ where the life is overestimated. Critically, however, as well as predicting the average life, the stochastic distribution of the MC simulated data is similar to that of the test data. It is very significant that the variation in fatigue response that arises due to the heterogenous microstructure can indeed be predicted by explicitly modelling the microstructural features.

The life predictions of the $\mathrm{R}=-1$ fatigue data are lower, by a factor of $2-3$, than the test data. The reasons for this are unclear but the model is over-predicting the effect of the compressive loading experienced in the $\mathrm{R}=-1$ cases. Given the accuracy of the $\mathrm{R}=0.1$ predictions and the use of a fundamental single parameter fatigue model it is perhaps too much of a simplification to expect it to collapse all R-ratio effects. The most likely reason for the under-prediction of the $\mathrm{R}=-1$ data, in comparison to $\mathrm{R}=0.1$, is the effect of the short crack growth phase between the predicted nucleation event and the actual development of an engineering crack which is represented by the test data. In the $\mathrm{R}=-1$ tests some of the crack growth will occur under compression when the crack faces are closed which is likely to lead to a reduction in short crack growth rates relative to $\mathrm{R}=0.1$ data. This element of short crack growth is not reflected in the current model but is being developed in separate work. 


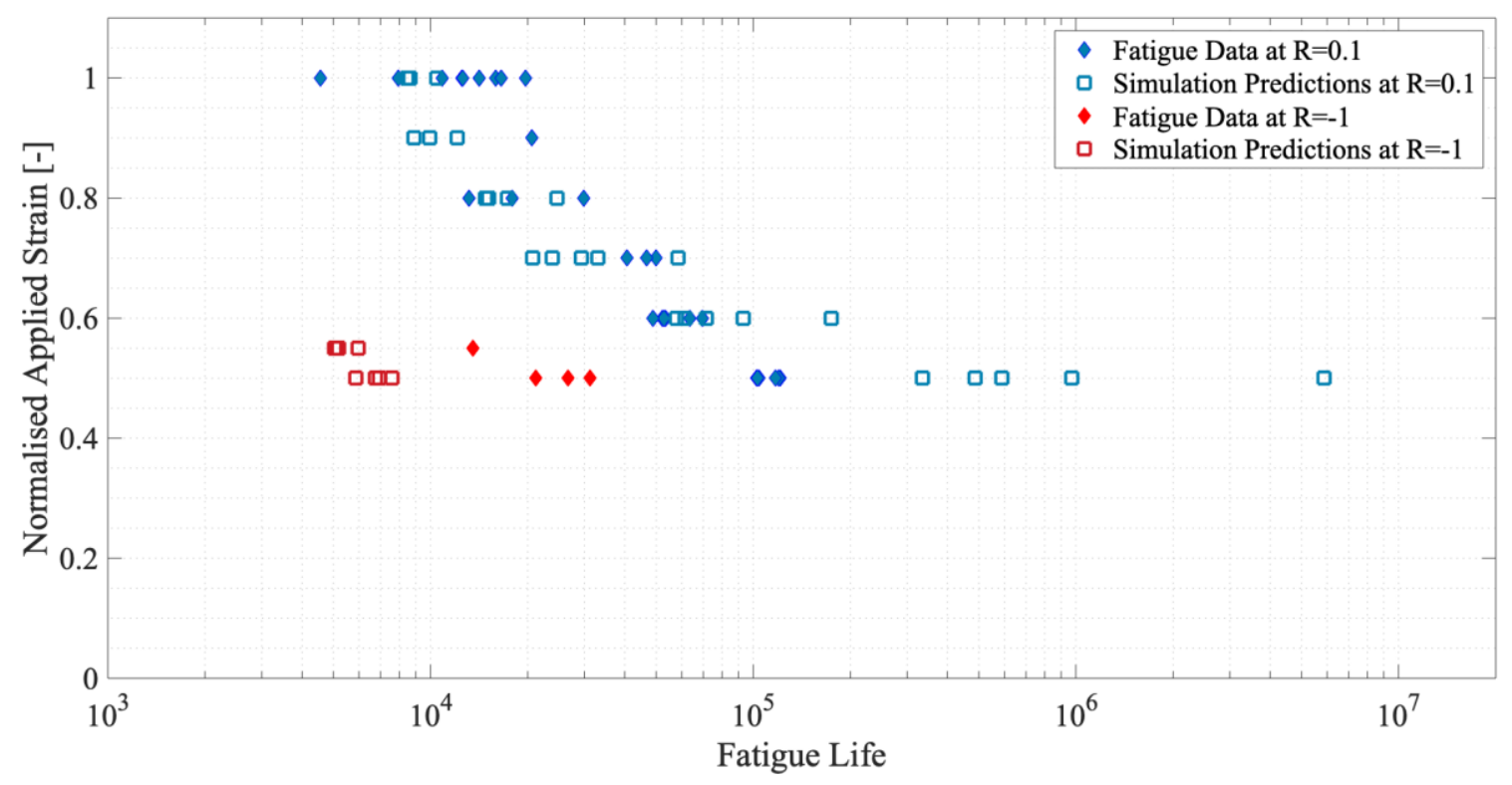

Figure 8. Predicted crack nucleation lives and experimental data for CG RR1000 for $\mathrm{R}=0.1$ and $\mathrm{R}=-1$ at $300^{\circ} \mathrm{C}$.

\subsection{CPFE Model Predictions at $700^{\circ} \mathrm{C}$}

Figure 9 shows comparisons of MC CPFE crack nucleation predictions with plain specimen fatigue data at $700^{\circ} \mathrm{C}$ and $\mathrm{R}=0.1$. The critical value of local stored energy required to nucleate a facet at $700^{\circ} \mathrm{C}$ is $3500 \mathrm{Jm}^{-2}$, as expected this is significantly less than the value of $60000 \mathrm{Jm}^{-2}$ obtained at $300^{\circ} \mathrm{C}$ reflecting the strong influence of temperature on fatigue life. Very good agreement can be seen between the predicted and simulated data, the shape of predicted and simulated S-N curves is matched well for a wide range of strain amplitudes and again the scatter in the MC data is very similar to the scatter in the experimental data. 


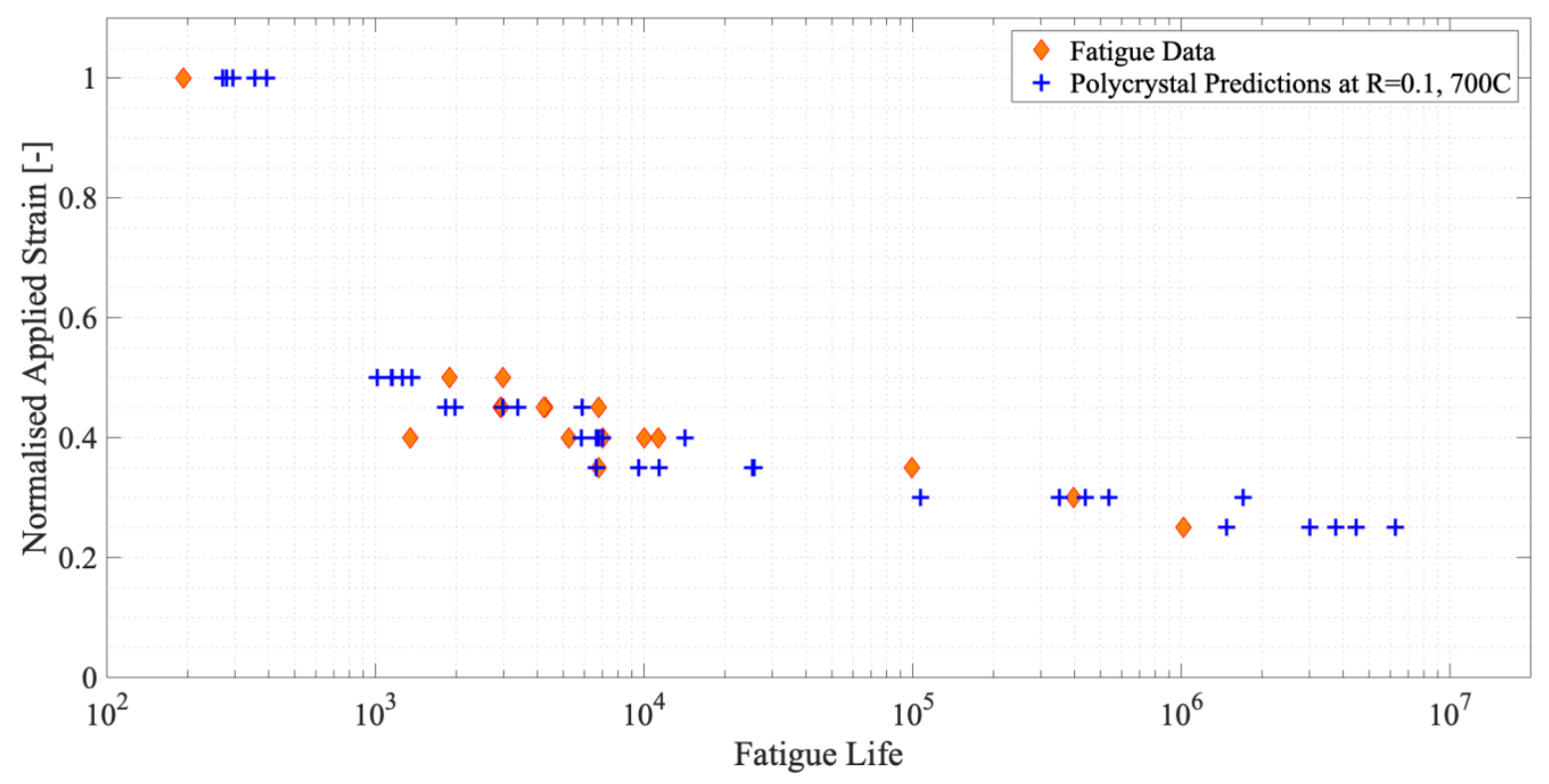

Figure 9. Comparison of predicted and experimental test data at $700^{\circ} \mathrm{C}$ and $\mathrm{R}=0.1$.

Figure 10 shows similar predictions and test data at $\mathrm{R}=-1$ using the same critical stored energy of $3500 \mathrm{Jm}^{-2}$. Whilst the gradient and scatter are predicted reasonably well, the majority of the data are under-predicted by a factor of approximately three. This is thought to be for the same reason as with the $300^{\circ} \mathrm{C}$ data that the compressive component of loading is not as damaging for the short crack growth phase and as this mechanism is not reflected in the current approach the test data show longer lives than the model predicts.

Another strong feature of the data in figure 10 is that at the highest strain range tested the test data are significantly lower than the predictions. Whilst there is only a single test point at this condition in figure 10 we have found this to be a consistent trend that the model over-predicts $\mathrm{R}=-1$ data at high strain ranges and high temperatures. This is thought to be due to a change in failure mechanism that changes from grain dominated failure at lower temperatures to inclusion dominated failure at higher temperatures and strain levels. The next section describes the effect of an embedded isolated inclusion on CPFE micro-mechanical results and in section 3.4 the effects of the inclusion on the fatigue predictions is shown. 


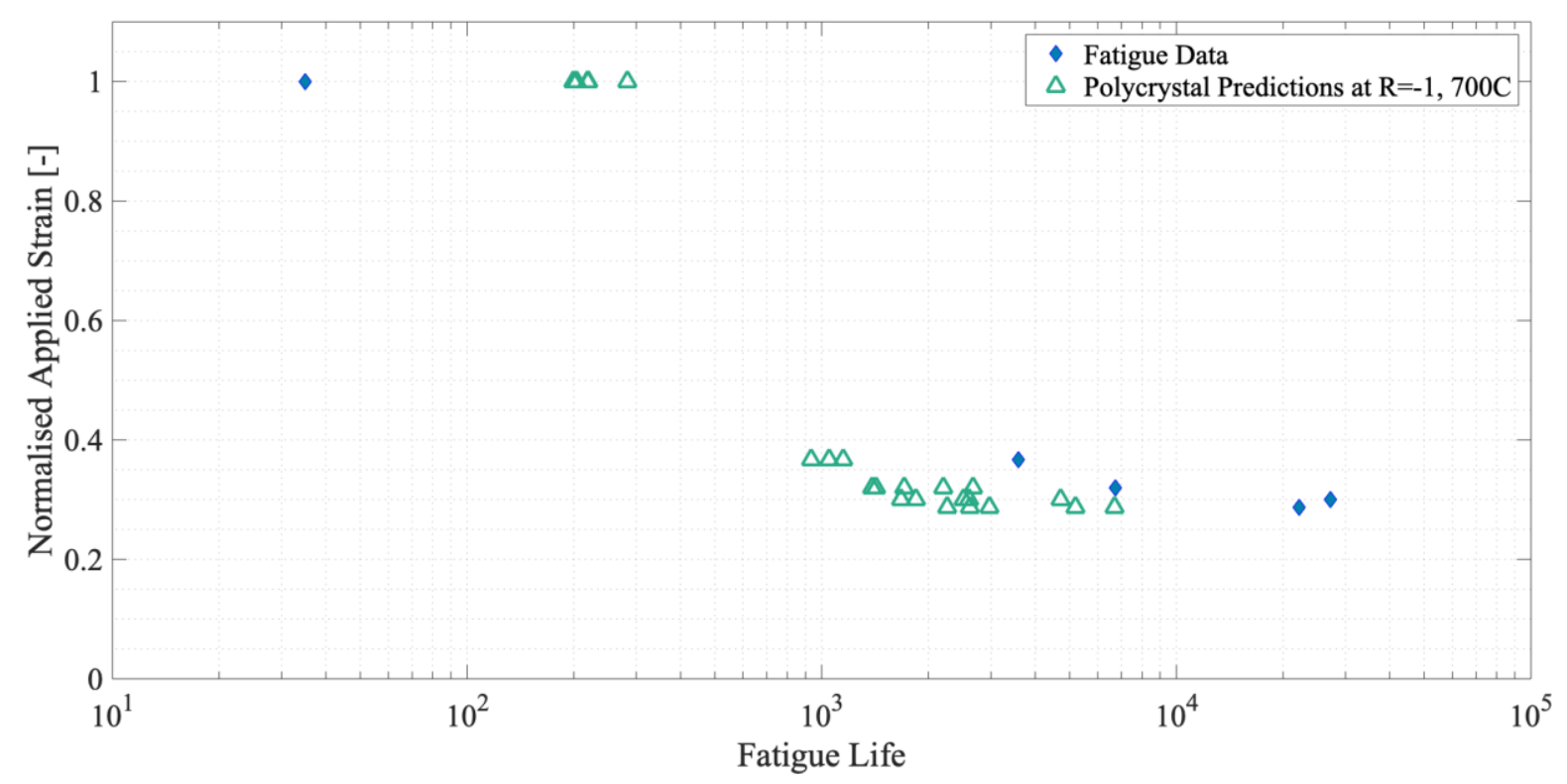

Figure 10. Predicted crack nucleation lives and experimental data at $700^{\circ} \mathrm{C}$ and $\mathrm{R}=-1$.

\subsection{Effect of Inclusions on Stress Redistribution and Life Prediction at $700^{\circ} \mathrm{C}$}

It is known that at high temperature in powder metallurgy alloys and specifically in Coarse Grain RR1000 that failure frequently occurs at inclusions embedded in the alloy matrix. There are many different types of possible inclusion that can form, including different compositions and morphologies. There are three ways in which an inclusion can result in initial crack nucleation, Bergsmo and Dunne ${ }^{37}$, i) fracture of the particle itself, ii) decohesion of the particle-matrix interface, and iii) facet nucleation in the matrix surrounding the particle. In any of these cases the initial crack in the particle or matrix may or may not lead to growth of the facet into a fatigue crack, for instance if the particle cracks it may not lead to the development of further crack growth within the matrix. It is interesting to note that this type of behavior is also found in different structures, Bellini et $\mathrm{al}^{39}$ in nodular Ductile Cast Iron, found similar mechanisms of nodule cracking, interface debonding and matix slip band crack initiation. The dominance of different mechanisms was dependent on ferritic or pearlitic microstructure and load levels.

Bandyopadhyay and Sangid ${ }^{40}$ performed an extensive study on the effect of different inclusion properties on driving forces for nucleation and preferential failure modes in the same alloy investigated here. Comparing inclusions with stiffness values approximately 50\% lower and 50\% higher than the stiffness of the matrix they found a stiffer inclusion would prefer an inclusion driven failure at any load condition and that the more compliant inclusion showed reduced propensity for 
inclusion driven failure, but also greater likelihood of inclusion failure in the HCF regime. In our investigation the stiffness of the inclusion is approximately twice that of the matrix which will prefer an inclusion driven failure mechanism. Bandyopadhyay and Sangid ${ }^{40}$ also studied the effect of different coefficients of thermal expansion (CTE) between the matrix and inclusion. At low strain ranges $(0.3 \%)$ a higher CTE in the inclusion resulted in a significant increase in local plastic strain close to the inclusion, but at higher strain ranges $(1 \%)$ the effect of different CTE was greatly reduced.

To quantify the effect of an inclusion on nucleation life in our CPFE studies, and to determine if this mechanism is responsible for the loss in accuracy in the model at $700^{\circ} \mathrm{C}$ and $\mathrm{R}=-1$, an oxide inclusion is embedded in the granular microstructure and its effect on the development of stresses and strains in the surrounding grains is investigated. The contact between the particle and alloy grains is modelled using the Cohesive Zone Method (CZM) described in section 2.4.

Figure 11(a) shows a section through a typical polycrystalline RVE used to represent CG RR1000 in the CPFE analyses. The specific RVE shown in figure 11 was used for a simulation at $700^{\circ} \mathrm{C}, \mathrm{R}=-1$ at the highest strain range. For comparison the stresses, strains and local energy in the absence of an inclusion are shown in figures 11(b-d). These images are all taken at the point of maximum strain on the $5^{\text {th }}$ loading cycle. In the absence of an inclusion the variations in the contour quantities arise out of differences in the properties of the grains in different orientations. The critical failure location is the point of maximum local stored energy shown in figure 12(b). As described in section 2.4, to insert an inclusion, a grain at the center of the RVE is simply reassigned with the properties of the oxide. The highlighted grain in figure 11(a) has therefore been converted to elastic isotropic oxide material.

Figure 12(a)-(b) shows identical contour quantities to figure 11(b)-(c), plotted at the same time point in the load history, for the RVE with an inclusion. The presence of the inclusion clearly increases the localization of energy, strain and stress in the surrounding grains. Under the high loading seen in this model the CZM surface between particle and matrix opens causing further stress redistribution and concentration thus increasing plasticity in the matrix at the edges of the opening of the inclusion. This interface failure also generates a noticeable relaxation of stress and strain along the cracked face of the inclusion. The interface initially opens over the first load cycle and is fully open after five cycles suggesting that actual nucleation life might be much lower than that calculated through the mechanism of local stored energy. That is, this de-cohesion process may be responsible for the test lives at high strain ranges being much lower than the predicted lives in figure 10. 


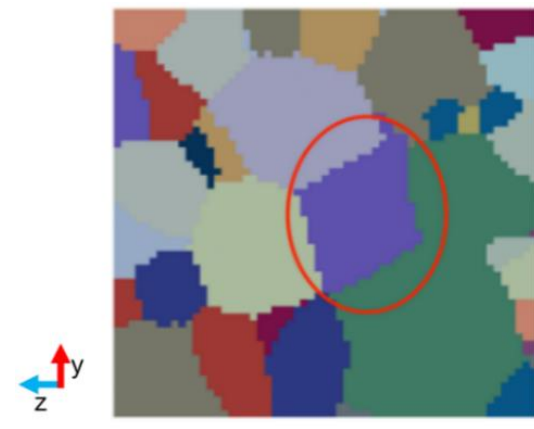

(a)

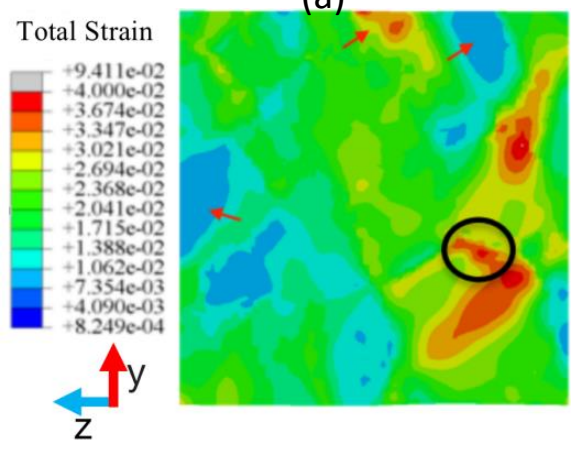

(c)

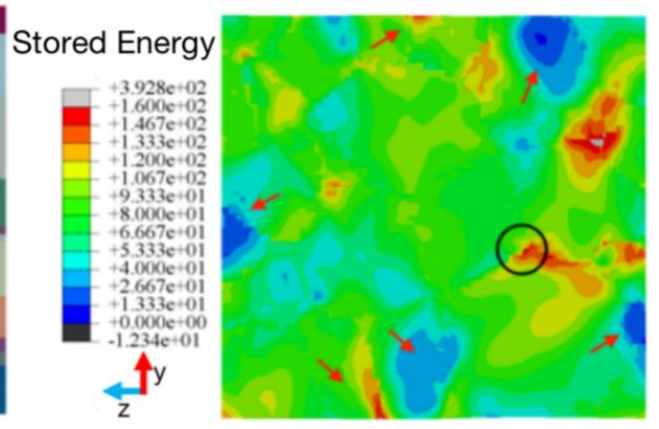

(b)
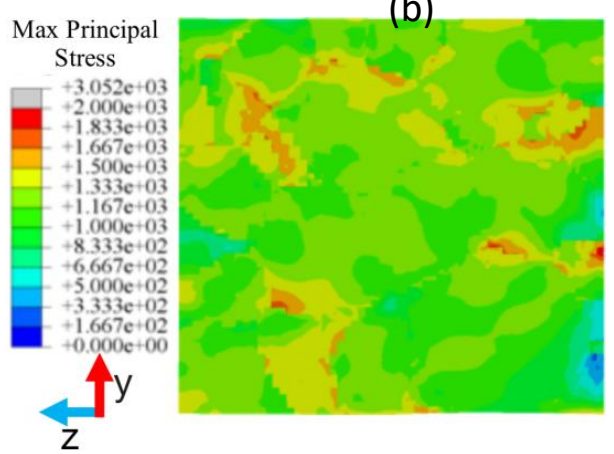

(d)

Figure 11. Microstructural RVE at maximum strain condition (in the horizontal in-plane Zdirection at $700^{\circ} \mathrm{C}$, (a) grain morphology, (b) total stored energy, (c) total strain in the load direction, (d) maximum principal stress $(\mathrm{MPa})$.

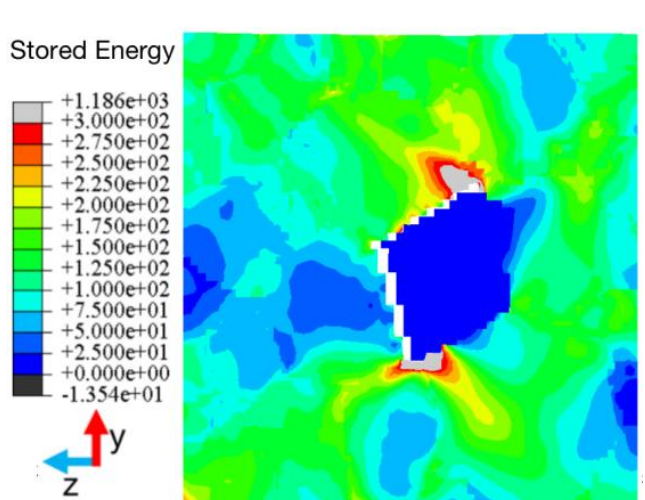

(a)

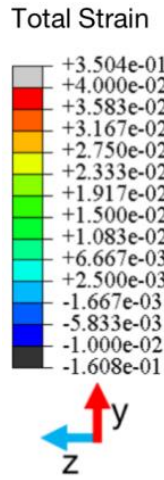

Figure 12. Inclusion based model showing (a) local stored energy (b) total strain in the load direction.

Figure 13 shows the comparison between test data at $700^{\circ} \mathrm{C} \mathrm{R}=-1$, and life predicted from the $\mathrm{CPFE}$ models with embedded inclusions. The life to crack nucleation is calculated exactly as before and as described in section 2.5 but the critical location is at the region ahead of the crack/decohered 
inclusion. The local energy accumulation will therefore be altered due to the presence of the inclusions and the debonded surface. The effect of the inclusion and debonding lowers the life of the high strain range tests due to the higher local stored energies. However, as is clear from figure 13 this reduction is not sufficient to match the actual test data, so whilst the CZM/inclusion model is able to predict interface decohesion and subsequent increase of local stress and strain due to the resulting stress concentration, the magnitude of this concentration does not correlate with the test data. At the lower strain magnitudes there is no interface failure and the predicted lives are similar to those without the inclusion. The reason that the high strain loading point in figure 13 is still not predicted by the model may be due to the fact that after interface failure the primary failure mechanism is of short crack propagation which, as has been discussed already is not captured by the current approach.

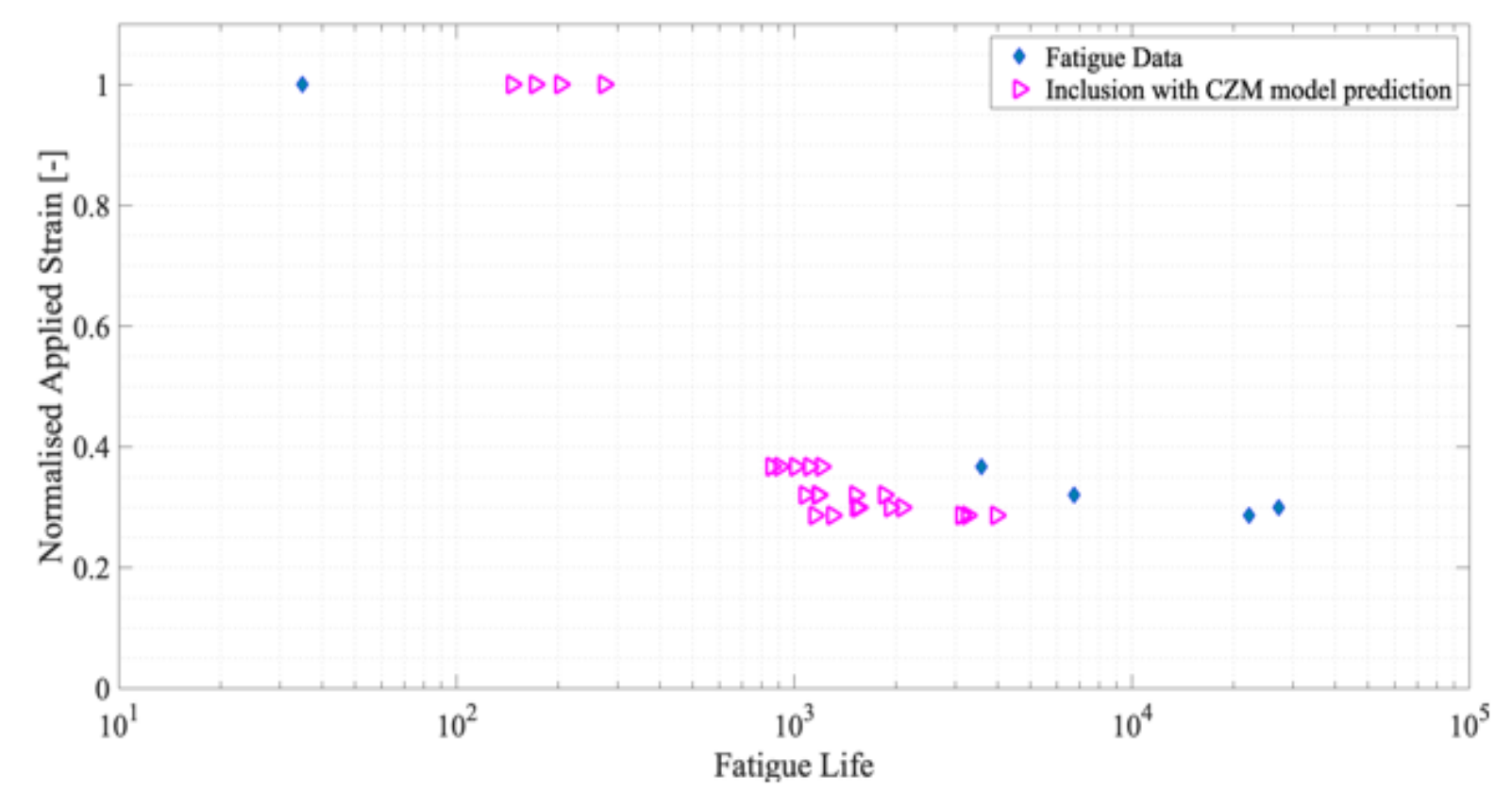

Figure 13. Simulated crack nucleation lives and experimental data for inclusion based model at $\mathrm{R}=-1$.

\subsection{Comparison of Simulated and Experimental Fatigue Distributions}

A key objective of mechanistic modelling of fatigue is to predict distributions and ideally tails of fatigue life. Predicting single points of fatigue test data is of limited use as critical components are 
invariably assessed to a certain probability of failure and so models that predict only the average response need to have minimum factors applied. These are usually derived from data necessitating a large amount of testing. Microstructural modelling lends itself to the determination of fatigue distributions as different representative microstructural instantiations can easily be generated and the results compared to fatigue data. Comparisons of the distributions of test data and simulated data are presented in this section.

As the number of data points at any given condition (both experimental and simulated) is quite small the entire data set at a given temperature and R-ratio is normalized to generate a larger dataset from which a more accurate standard deviation can be calculated. This normalization is done by taking the average of the data (in log space) at each condition, and then determining the deviation between each data point and the mean at that condition. The combined set of deviations can then be used to determine a population standard deviation. It is acknowledged that this assumes the standard deviation itself does not change with strain level, the only recourse to this problem is to run more simulations and generate more test data. This process is performed separately for the test data and the simulated data. Using this method figure 14 plots the Cumulative Distribution Functions (CDFs) generated entirely from the simulated data and compares them to test data, (a) is for data at $300^{\circ} \mathrm{C}$ and (b) for $700^{\circ} \mathrm{C}$. Distributions were only generated for the $\mathrm{R}=0.1$ data as $\mathrm{R}=-1$ showed systematic differences between test data and simulation. The failure probability for the test data was calculated by normalizing the entire data set (at each temperature) and ranking each point based on its deviation from the mean.

The graphs in figure 14 were created in the following manner for results at a given temperature and R-ratio:

A. For the simulated CDFs

a. $\log (10)$ of each simulated life was calculated.

b. The mean of the log lives was calculated at each individual test condition.

c. The ratio between each simulated $(\log )$ life to the mean $(\log )$ life at each specific test condition was obtained.

d. The total set of ratios obtained from (c.) represents the normalized variation of the entire population, the standard deviation was taken from the values obtained from (c.). 
e. Using the standard deviation from (d.) and the mean of the simulated data at each condition, CDF curves for each condition were generated as plotted by the solid lines in figures 14 (a) and (b).

\section{B. For the test data CDFs}

a. $\log (10)$ of each test life was calculated.

b. The ratios between each test $(\log )$ life point and the mean of the simulated data at that condition was calculated.

c. The set of data obtained from (b.) was ranked in numerical order and the rankings used to determine the probability of failure for each test. ie for a data set of ' $n$ ' samples a test ranked ' $\mathrm{x}$ ' in the population will have a cumulative probability of failure of $x /(n+1)$.

d. The probabilities of failure for the test data obtained from (c.) and the respective lives are plotted as the solid points in figures 14 (a) and (b).

There is still work to be done in developing the accuracy of this method over the complete range of data but the results clearly indicate the potential to virtually simulate fatigue distributions. It is remarkable that it is possible to simulate full distributions of fatigue life over ranges of conditions using a single parameter (the critical stored local energy). A greater number of both test and simulated data is still required however to determine accurately the tails of the distributions.

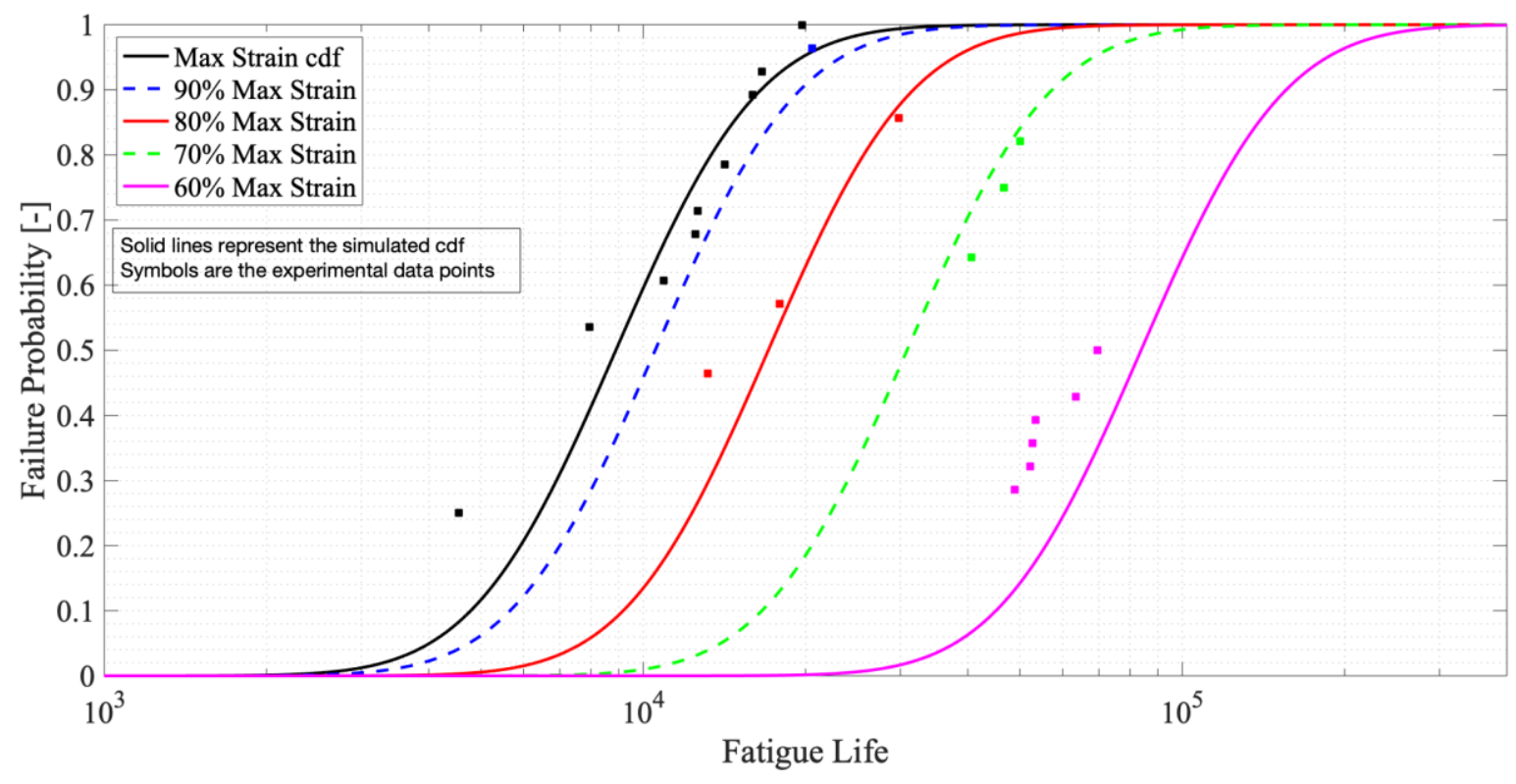

(a) 


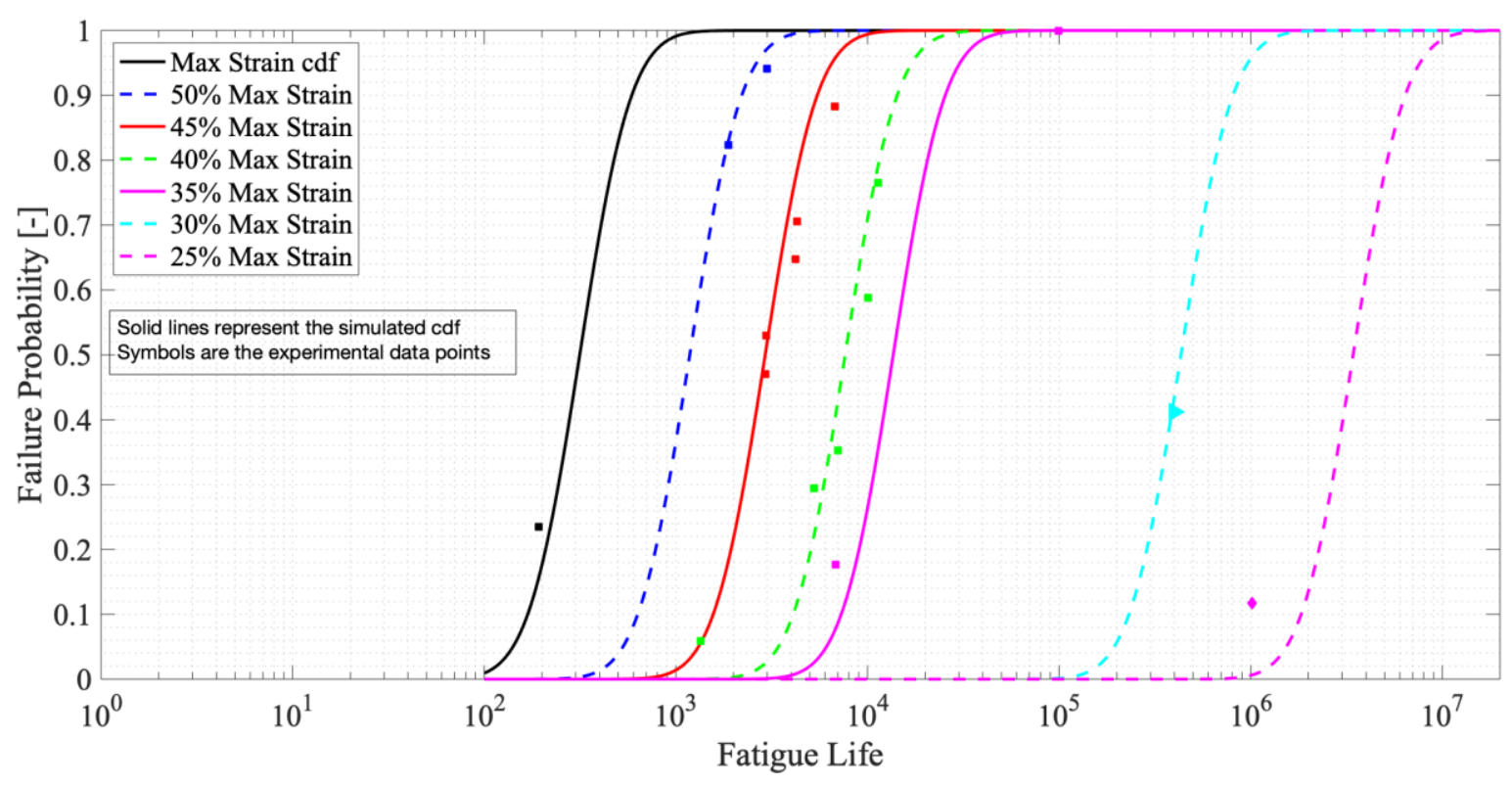

(b)

Figure 14: Comparisons of simulated and experimental CDF plots, (a) $300^{\circ} \mathrm{C} \mathrm{R}=0.1$ data (b) $700^{\circ} \mathrm{C} \mathrm{R}=0.1$ data

\section{Conclusions}

1. A stochastic MC CPFE approach using local stored energy has been used to simulate fatigue crack nucleation in CG RR1000 at $300^{\circ} \mathrm{C}$ and $700^{\circ} \mathrm{C}$ and for R-ratios of 0.1 and -1 . Critical local stored energies of $60000 \mathrm{Jm}^{-2}$ and $3500 \mathrm{Jm}^{-2}$ were determined for temperatures of 300 and $700^{\circ} \mathrm{C}$ respectively.

2. At both temperatures the $\mathrm{R}=0.1 \mathrm{MC}$ CPFE predictions capture the mean, slope and distribution of experimental fatigue test data. This is extremely encouraging as it suggests explicit microstructural modelling can be used to virtually simulate distributions of fatigue test data.

3. The data at $\mathrm{R}=-1$ were under-predicted by the model by a factor of $2-3$. The reason for this is thought to be that the actual fatigue failures include a component of short crack growth, after facet nucleation and prior to specimen failure. For the $\mathrm{R}=-1$ tests, as part of the load cycle is in compression, the crack growth rates are likely to be lower than for the $\mathrm{R}=0.1$ tests (for the same strain range) leading to the conservative predictions. A model is currently being developed to simulate SCG explicitly in a CPFE model. 
4. At $700^{\circ} \mathrm{C}$ failure is frequently nucleated at inclusions embedded in the alloy matrix, this mechanism has been included in the CPFE modelling using a cohesive zone to define the interface between inclusion and alloy. At high applied strains decohesion of the interface is predicted which leads to lower failure lives. The model predicts a greater localization of stored energy in the alloy surrounding the inclusion due to this decohesion but this greater energy does not correlate with the observed reduction in fatigue life.

5. The research highlights the current capabilities of CPFE used in conjunction with the local stored energy to predict fatigue nucleation life for a range of fatigue data. The use of an MC CPFE approach is a viable method to simulate distributions of fatigue data. Further work is required to develop a SCG modelling capability which may explain some of the differences between the test data and simulated predictions.

\section{Acknowledgements}

The authors are grateful to Imperial College London for the provision of research facilities for this work, to the Royal Society for financial support under Industry Fellowship grant IF170004, as well as to Rolls-Royce plc for discussions and provision of fatigue test data.

\section{Bibliography}

[1]. Wan VV, MacLachlan DW, Dunne FP. A stored energy criterion for fatigue crack nucleation in polycrystals. Int J Fatigue. 2014; 68: 90-102.

[2]. Wan VV, Jiang J, MacLachlan DW, Dunne FP. Microstructure-sensitive fatigue crack nucleation in a polycrystalline Ni superalloy. Int J Fatigue. 2016; 90: 181-190.

[3]. Groeber MA, Jackson MA. DREAM 3D: a digital representation environment for the analysis of microstructure in 3D. Integrating materials and manufacturing innovation. 2014.

[4]. Chen B, Jiang J, Dunne FP. Microstructurally-sensitive fatigue crack nucleation in Ni-based single and oligo crystals. J. Mech. Phys. Solids. 2017; 106: 15-33.

[5]. Chen B, Jiang J, Dunne FP. Is stored energy density the primary meso-scale mechanistic driver for fatigue crack nucleation? Int. J. Plasticity. 2018; 101: 213-229.

[6]. McDowell DL, Dunne FP. Microstructure-sensitive computational modeling of fatigue crack formation. Int. J. Fatigue. 2010; 9: 01521-42. 
[7]. Sauzay M, Jourdan T. Polycrystalline microstructure, cubic elasticity, and nucleation of highcycle fatigue cracks. Int. J. Fracture. 2006; 141(3-4): 431-46.

[8]. Sangid MD, Maier H, Sehitoglu H. An energy based microsturcture model to account for fatigue scatter in polycrystals. J. Mech. and Phys. of Solids. 2011; 59: 595-609.

[9]. Sangid MD, Sehitoglu H, Maier H, Niendorf T. Grain boundary characteristics and energetics of superalloys. Mat. Sci. Eng. A. 2010; 527: 7115-7125.

[10]. Sangid MD, Ezaz T, Sehitoglu H, Robertson IM, Energy of slip transmission and nucleation at grain boundaries. Acta Mat. 2011; 59: 283-296.

[11]. Stopka KS, McDowell DL. Microstructure sensitive computational multiaxial fatigue of AL 7075-T6 and duplex Ti-6Al-4V. Int. J. Fatigue. 2020;133.

[12]. Tang Gu, Stopka KS, Xu C. McDowell DL, Prediction of maximum fatigue indicator parameters for duplex Ti-6Al-4V using extreme value theory. Acta Mat. 2020; 188: 504-516.

[13]. Gillner K, Munstermann S. Numerically predicted high cycle fatigue properties through representative volume elements of the microstructure. Int. J. Fatigue. 2017; 105: 219-234.

[14]. Owolabi GM, Whitworth HA. Modelling and simulation of microstructurally small crack formation and growth in notched nickel-base superalloy component. J. Mater. Sci. Tech. 2014; 30(3): 203-212.

[15]. Mughrabi H. The cyclic hardening and saturation behaviour of copper single crystals. Materials Science and Engineering. 1978; 33(2): 207-223.

[16]. Dunne FP, Wilkinson AJ, Allen R. Experimental and computational studies of low cycle fatigue crack nucleation in a polycrystal. Int. J. Plasticity. 2007; 23(2): 273-295.

[17]. Repetto EA, Ortiz M. A micromechanical model of cyclic deformation and fatigue-crack nucleation in fcc single crystals. Acta Mat. 1997; 45(6): 2577-2595.

[18]. Shanthraj P, Zikry MA. Microstructurally induced fracture nucleation and propagation in martensitic steels. J. Mech. Phys. of Solids. 2013; 61(4): 1091-1105.

[19]. Mughrabi H. Cyclic slip irreversibility and fatigue life: A microstructure-based analysis. Acta Mat. 2013; 61(4): 1197-203.

[20]. Manonukul A, Dunne FP. High-and low-cycle fatigue crack initiation using polycrystal plasticity. Proc. Royal Society of London. A: Mathematical, Physical and Engineering Sciences. 2004; 460(2047): 1881-1903.

[21]. Zheng Z, Prastiti NG, Balint DS, Dunne FPE. The dislocation configurational energy density in discrete dislocation plasticity. J. Mech. Phys. of Solids. 2019; 129: 39-60. 
[22]. Prastiti NG, Xu Y, Balint DS, Dunne FPE. Discrete dislocation, crystal plasticity and experimental studies of fatigue crack nucleation in single-crystal nickel. Int. J. Plasticity, $2020 ; 126$.

[23]. Lavenstein S, El-Awady JA. Micro-scale fatigue mechanisms in metals: Insights gained from small-scale experiments and discrete dislocation dynamics simulations. Current opinion in solid state \& materials science. 2019; 23.

[24]. Zheng Z, Stapleton A, Fox K, Dunne FPE. Understanding thermal alleviation in cold dwell fatigue in titanium alloys. Int. J. Plasticity. 2018; 111: 234-252.

[25]. Fatemi A, Socie DF. A critical plane approach to multiaxial fatigue damage including out-ofphase loading. Fatigue \& Fracture of Engineering Materials \& Structures. 1988; 11(3):149165.

[26]. Dong-Feng L, Barrett RA, O'Donoghue PE, Hyde CJ, O'Dowd NP, Leen SB. Micromechanical finite element modelling of thermo-mechanical fatigue for P91 steels. Int. J. Fatigue, 2016; 87: 192-202.

[27]. Yuan G-J, Zhang X-C, Chen B, Tu S-T, Zhang C-C, Low-cycle fatigue life prediction of a polycrystalline nickel base superalloy using crystal plasticity modelling approach. J. Mat. Sci. \& Tech, 2020; 38:28-38.

[28]. Yuan G-J, Wang R-Z, Gong C-Y, Zhang X-C, Tu S-T, Investigation of micro-notch effect on small fatigue crack behaviour in nickel-based alloy GH4169: Experiments and Simulations. Int. J. Fatigue, 2020; 136:

[29]. Dabiri M, Lindroos M, Andersson R, Afkhami S, Laukkanen A, Bjork T. Utilizing the theory of critical distances in conjunction with crystal plasticity for low-cycle notch fatigue analysis of S960 MC high-strength steel. Int. J. Fatigue 2018; 117: 257-273.

[30]. Musinski WD, McDowell DL. Microstructure-sensitive probabilistic modelling of HCF crack initiation and early crack growth in Ni-base superalloy IN100 notched components. Int. J. Fatigue. 2012; 37: 41-53.

[31]. Bridier F, McDowell DL, Villechaise P, Mendez J. Crystal plasticity modelling of slip activity in Ti-6Al-4V under high cycle fatigue loading. Int. J. Plasticity. 2009; 25: 10661082.

[32]. Khan AS, Huang S. Continuum theory of plasticity. John Wiley \& Sons, Inc 1995. ISBN 0471-31043-3.

[33]. Zhang T, Jiang J, Shollock BA, Britton TB, Dunne FP. Slip localization and fatigue crack nucleation near a non-metallic inclusion in polycrystalline nickel-based superalloy. Materials Science and Engineering: A. 2015; 641: pp 328-39. 
[34]. Texier D, Cormier J, Villechaise P, Stinville JC, Torbet CJ, Pierret S, Pollock TM. Crack initiation sensitivity of wrought direct aged alloy 718 in the very high cycle fatigue regime: the role of non-metallic inclusions. Materials Science and Engineering: A. 2016; 678: 122-36.

[35]. Yeratapally SR, Glavicic MG, Hardy M, Sangid MD. Microstructure based fatigue life prediction framework for polycrystalline nickel-base superalloys with emphasis on the role played by twin boundaries in crack initiation. Acta Materialia 2016; 107: 152-167.

[36]. Papoulia KD, Vavasis SA, Sam C, Ganguly P. Analysis of mesh dependence in rigid cohesive interface finite element models. 11th International Conference on Fracture 2005; 1235-1240.

[37]. Bergsmo A, Dunne FP. Competing mechanisms of particle fracture, decohesion and slipdriven fatigue crack nucleation in a PM Nickel superalloy. Int. J. of Fatigue. 2020; 135: 105573.

[38]. Gao YF, Bower AF. A simple technique for avoiding convergence problems in finite element simulations of crack nucleation and growth on cohesive interfaces. Modelling and Simulation in Materials Science and Engineering. 2004; 12(3): 453.

[39]. Bellini C, Di Cocco V, Favaro G, Iacoviello F, Sorrentino L. Ductile cast irons: Microstructure influence on the fatigue initiation mechanisms. Fatigue \& Fracture of Engineering Materials \& Structures. 2019; 42(9): 2172-2182.

[40]. Bandyopadhyay R, Sangid MD. Crystal plasticity assessment of inclusion- and matrix-driven competing failure models in a nickel-base superalloy. Acta Materialia. 2019; 177: 20-34. 